\title{
Lithographically defined nickel wires for gasflow sensing applications
}

\author{
Sebastian Quednau, Helmut F. Schlaak, \\ Technische Universität Darmstadt, Institute for Electromechanical Design \\ s.quednau@emk.tu-darmstadt.de
}

\begin{abstract}
:
This work introduces a process for the fabrication of freely suspended nickel wires with at least one dimension smaller than one micron. The wires are fabricated by photolithography with subsequent electrodeposition. They are used as temperature sensing elements in a calorimetric gas flow sensor and have a high potential of reducing the response time of such devices. A fabricated sensor element was placed in a gas flow channel to carry out a steady state characterization and to prove its functionality. The thermal coefficient of electrical resistance was determined by measurements in a climate chamber. Additionally we carried out a CFD calculation in order to determine the optimal sensor element design.
\end{abstract}

Key words: MEMS fabrication process, electrodeposition, gas flow sensor, CFD

\section{Introduction}

Thermal gas flow sensors are used in a wide variety of applications such as air flow controlling in fuel engines. There are principally three different configurations for micromachined thermal gas flow sensors [1], [2]:

$$
\begin{aligned}
& \text { 1. hot wire or hot film anemometers } \\
& \text { 2. calorimetric measuring devices } \\
& \text { 3. time of flight sensors }
\end{aligned}
$$

Calorimetric gas flow sensors use separate heaters and temperature sensing elements. Usually one temperature sensing element is placed in front of (upstream) and behind (downstream) the sensor. When the gas is at rest, there is a symmetric heat distribution around the central heating element. When a gas flow occurs the temperature field becomes asymmetric, thus both temperature measuring elements detect different temperatures. The advantage is the high sensitivity at small gas flow velocities. The disadvantage is the longer response time due to changes in the gas flow velocity. Since the calorimetric device uses two temperature sensing elements the direction of the gas flow can be predicted and there is no need to integrate an additional reference temperature sensor. The third concept is the generation of a fast heat pulse by a thin heating wire. The configuration of the wires is the same as for the calorimetric sensor: a heating wire is placed between to measuring wires. The transit time of the heat pulse is measured and depends on the velocity of the gas flow. The three operational principles of thermal gas flow sensors are shown in Tab. 1.

In most applications the resistance of the wires is measured to determine its temperature. Since MEMS technologies provide the fabrication of thin films the wires are mostly replaced by thin films deposited on membranes to improve their thermal insulation from the substrate. Thus MEMS flow sensors always measure the velocity in the boundary layer where the thermal transport and the fluid velocity are significantly smaller than in the channel center. This decreases the signal strength and increases the response time [3], [4].

In this paper we present a method, to fabricate gas flow sensors with freely suspended thin wires. Although all three working principles can be implemented with this process we decided to fabricate a calorimetric gas flow sensor. The heater is implemented by using conventional thin film techniques and only the temperature sensing wires are fabricated by the proposed process. In order to increase the response velocity of the gas flow sensor it is suitable to keep the diameter of the measuring wire as small as possible. The process presented in this paper enables the fabrication of thin wires with 
Tab. 1: Operating principles of thermal gas flow sensors

Hot wire anemometer - the gas flow increases the heat transport from the wire resulting in ether a lower temperature or higher power consumption

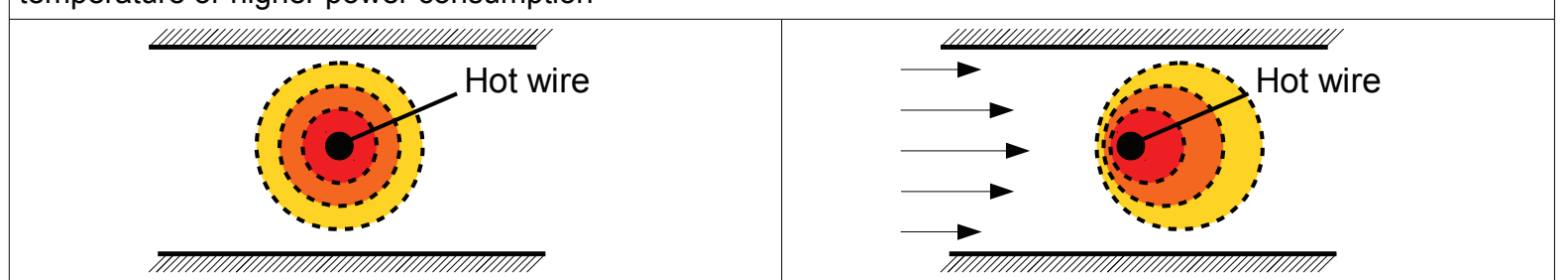

Calorimetric gas flow sensor - the gas flow shifts the temperature field downstream resulting in a different temperature at the up- and downstream measuring wire $\left(T_{2}>T_{1}\right)$

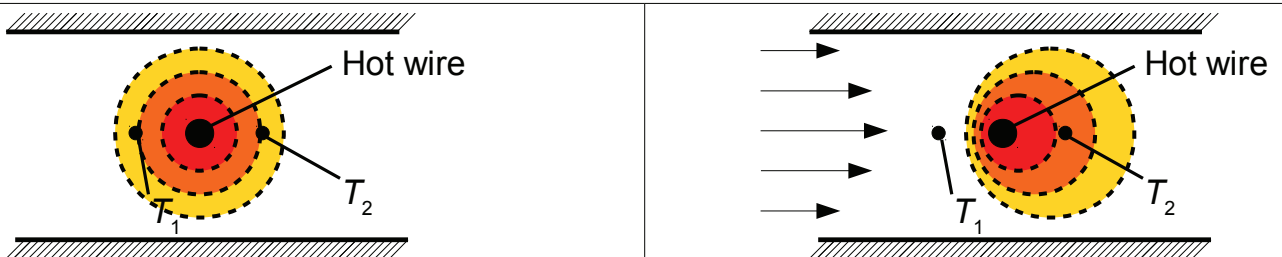

Time-of-flight sensor - a heat pulse generated by the hot wire is transported by the gas flow. The transition time to the downstream element is shorter than to the upstream element $\left(t_{2}<t_{1}\right)$.

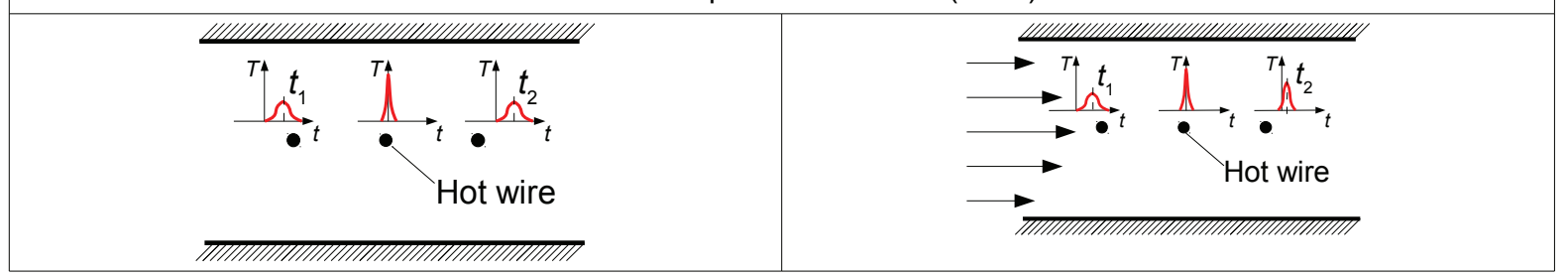

rectangular cross section and a width of $2 \mu \mathrm{m}$ and a height of $100-200 \mathrm{~nm}$. It has been shown, that flow sensors with such thin sensing wires can easily be used for photoacustic [5] or sound intensity measurements [6].

\section{Process for the fabrication of thin wires}

The developed process shown in Fig. 1 is adapted from [7]. The thin nickel wires are fabricated by electrodeposition on an etched step of a metallic sacrificial layer. The first step is to evaporate a chromium (20 nm) and nickel $(150 \mathrm{~nm})$ layer onto a borofloat ${ }^{\circledR}$ wafer. This layer is structured by photolithography in order to fabricate conducting paths like contact pads and the electrical thin film heater (Fig. 1 a)). To avoid corrosion and to enable wire bonding to connect the sensor with a socket electrically the metallic structures are coated with a thin gold layer by electroless chemical deposition. The next process step is the generation of a sacrificial layer (Fig. 1 b)). The thickness of the layer specifies the height in which the wires are spanned over the substrate. In our case, the sacrificial layer consists of $25 \mu \mathrm{m}$ SU-8 (SU-8 2025). It contains holes where the suspension of the wires is fabricated in later process steps. On the SU-8 layer a metallic layer (copper) is deposited by sputtering. The thickness of this layer specifies the thickness of the wires used as temperature measuring elements. The layer is structured to obtain islands of metal, which defines the position of the metal wires (Fig. 1 c)). A second photolithography and etching process defines the etched steps where the metallic wires are deposited (Fig. 1 d) and e)). The electrodeposition of the wires, without stripping the photoresist, is the crucial process step (Fig. 1 f)). As wire material we use nickel. Subsequent the photoresist is stripped (Fig. 1 g)) and the substrate is coated by a second copper layer which forms a seed layer for the subsequent electrodeposition of the suspensions (Fig. $1 \mathrm{~h}$ )). A layer of photoresist is placed on top in order to fabricate galvanic forms for the suspension of the nickel wires (Fig. 1 i)). The suspension is fabricated by electrodeposition of nickel (Figure $1 \mathrm{k})$ ). After depositing the suspension, the resist is stripped and the copper layer is removed (Fig. 1 I)). Then the chips can be diced. After dicing, the sacrificial SU-8 layer can be removed by oxygen plasma (Fig. $1 \mathrm{~m}$ )). During the dicing process the sensitive wires are protected by the SU-8 sacrificial layer. 
a)

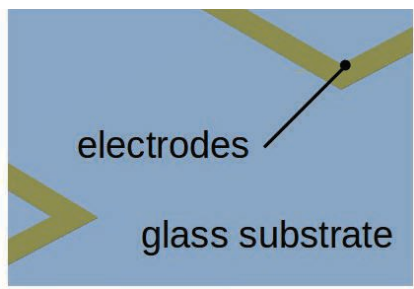

b)

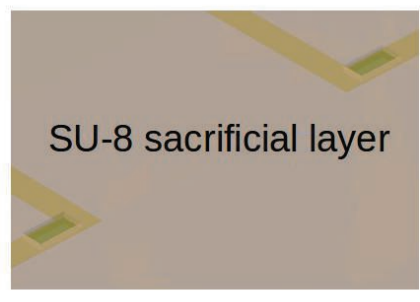

c)

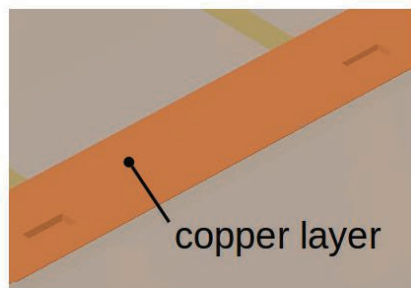

d)

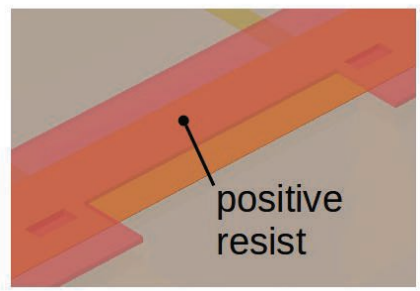

e)

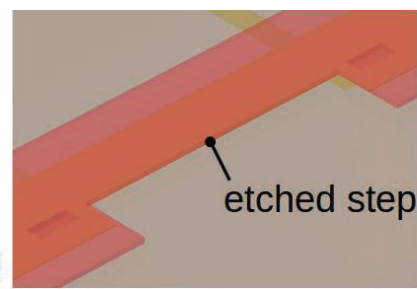

f)

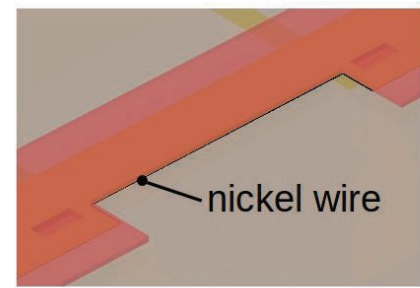

g)

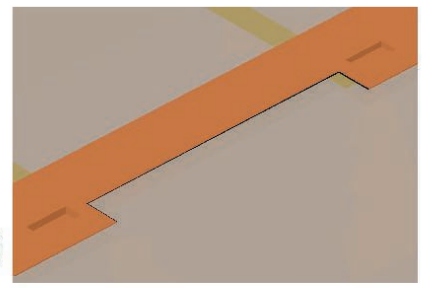

h)

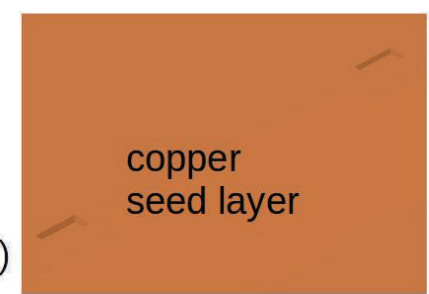

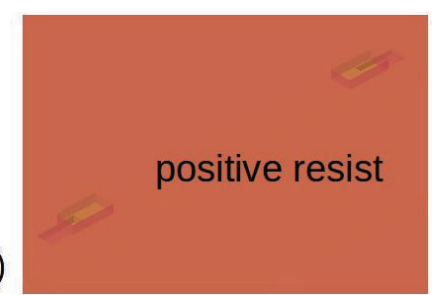

k)

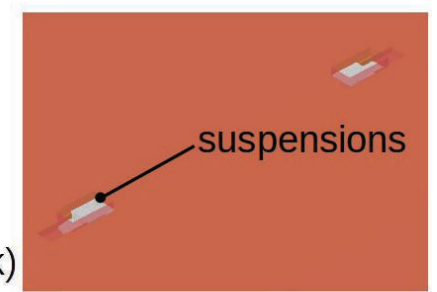

I)

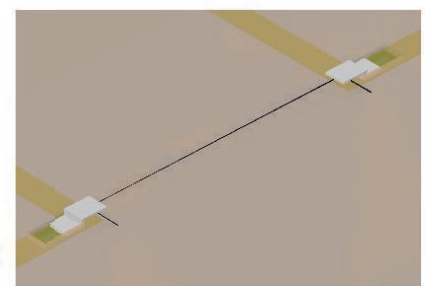

m)

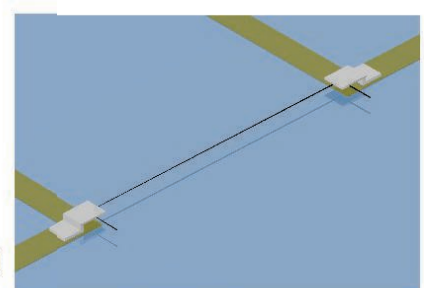

Fig. 1: Process flow for the fabrication of horizontal nickel nanowires by electro deposition of an etched step; a) Preparation of gold electrodes and conducting pathes on a glass substrate, b) coating and structuring a $25 \mu \mathrm{m}$ thick SU-8 sacrificial layer, c) sputtering and structuring a 100-200 nm thick copper layer, d) coating and structuring a positive photoresist layer, e) etching the copper film to prepare the etched step, f) electrodeposition of the nickel wire onto the prepared etched step, g) stripping the photo resist, $h$ ) sputtering a seed layer for the electrodeposition of the suspensions i) coating and structuring of positive photoresist to obtain galvanic forms to fabricate the suspensions, $k$ ) electrodeposition of the suspensions, I) stripping of the positive photoresist, removal of the copper film, $m$ ) removal of the SU-8 sacrificial layer by $\mathrm{O}_{2}$-plasma.

\section{Electrodeposition of the thin wires}

The electrodeposition of the wires (Figure $1 \mathrm{f}$ )) is the crucial step of the fabrication process. The cross section of the wire is shaped rectangular, since the etch process to fabricate the etched step causes under etching of the photoresist resulting in a rectangular cavity (Figure 2). So the thickness of the wire can be adjusted by varying the thickness of the copper layer on the SU-8 sacrificial layer. We chose a thickness of $200 \mathrm{~nm}$.

The electrodeposition of the wires is carried out in potentiostatic mode, since the real deposition area is unknown and varies during the process, especially when the cavity below the AZ 9260 photoresist is filled.
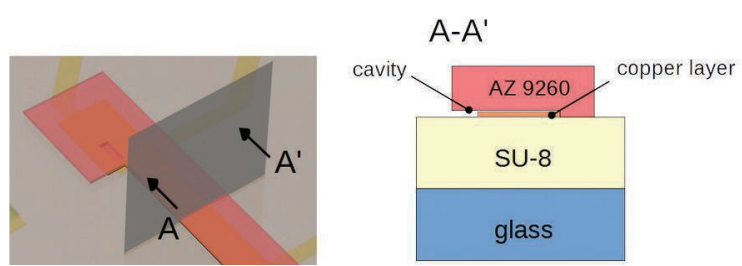

Fig. 2: Cavity caused by the under etching of the photoresist

We use a nickel sulfamate electrolyte to fabricate the wires. It enables stress free nickel structures and the deposition starts very reliably. Tab. 2 shows the parameters for the electrodeposition of the nickel wires. In order to ensure a completely wetting of the cavity, the wafer is kept wet after the etching of the copper 
layer. So the cavities are always filled with fluid and do not dry out.

Tab. 2: Electrolyte composition Process parameters and for the electrodeposition of the nickel wires

\begin{tabular}{|c|c|}
\hline $\begin{array}{l}\text { Electrolyte } \\
\text { composition }\end{array}$ & $\begin{array}{l}\text { Self-made electrolyte, } \\
\text { nickel sulfamate } \\
\left(\mathrm{Ni}^{-}\left(\mathrm{SO}_{3} \mathrm{NH}_{2}\right)_{2}\right): 275 \mathrm{~g} \mathrm{l}^{-1} \\
\text { boric acid }\left(\mathrm{H}_{3} \mathrm{BO}_{3}\right): 35 \mathrm{~g} \mathrm{l}^{-1}\end{array}$ \\
\hline \multirow{3}{*}{$\begin{array}{l}\text { Deposition } \\
\text { parameters }\end{array}$} & $\begin{array}{l}\text { Mode: Potentiostatic vs. } \\
\mathrm{Ag} / \mathrm{AgCl} \text { reference electrode }\end{array}$ \\
\hline & $\begin{array}{l}\text { Plate sequence: } 10 \mathrm{~ms} @ 0,9 \\
\mathrm{~V} ; 40 \mathrm{~ms} @ 0,35 \mathrm{~V}\end{array}$ \\
\hline & Bath temperature: $45^{\circ} \mathrm{C}$ \\
\hline
\end{tabular}

Fig. 3 shows an SEM image of the fabricated thin wire with a length of $20 \mu \mathrm{m}$ and a cross section of $200 \mathrm{~nm} \times 2 \mu \mathrm{m}$, which can be used as thermal measuring element in calorimetric gas flow devices.

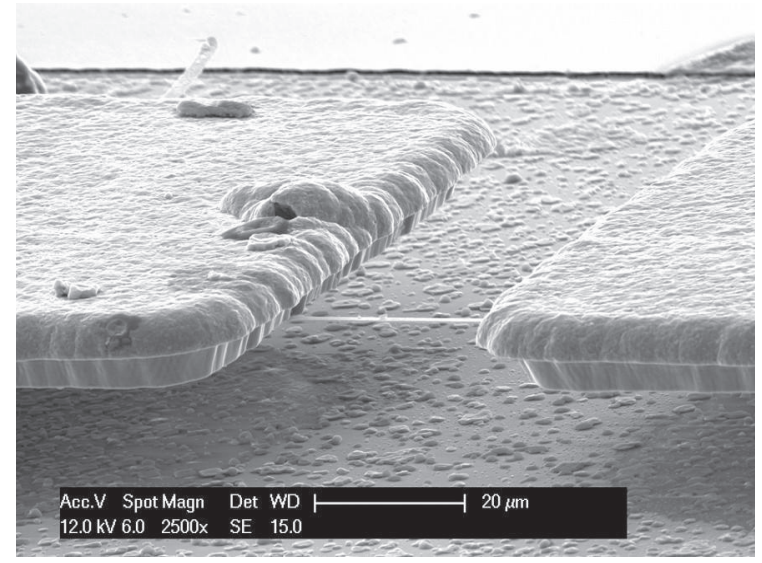

Fig. 3: Freely suspended thin nickel wire fabricated by electroplating

\section{Gasflow sensor with electrodeposited thin wires}

To fabricate a gas flow sensor, which uses etched step deposited nickel wires as sensitive elements it is necessary to connect them electrically. In order to characterize them every wire is connected by four conducting paths in order to enable four point measurement of resistance. So we can measure the resistance of the wire while the influence of the conducting paths is eliminated. By applying an alternating current to measure the resistance we can also eliminate the influence of thermal contact voltages in the system. We selected the calorimetric gas flow sensing principle for sensor fabrication according to Tab. 1. To simplify the process, only the temperature measuring elements are implemented by nickel wires. The heater is implemented as a thin film heater since it simplifies the electrical wiring and the fabrication process. The thin film heater can be driven in constant power mode since the high thermal mass of the chip ensures an almost constant heating temperature. The downstream and upstream measurement elements are implemented as thin nickel wires fabricated with the process described above.

Fig. 4 shows a SEM image of the realized gas flow sensor. The temperature sensing wires have a length of $200 \mu \mathrm{m}$ and a cross section of $200 \mathrm{~nm} \times 2 \mu \mathrm{m}$.

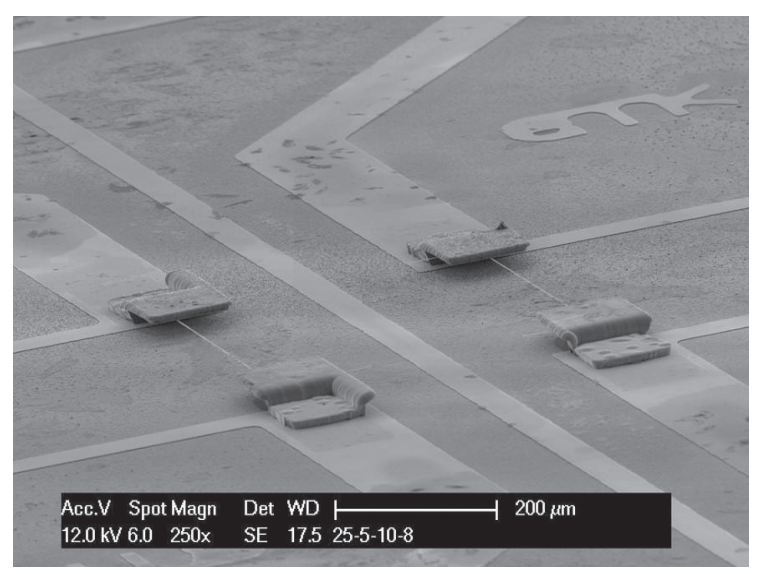

Fig. 4: SEM image of the fabricated calorimetric gas flow sensor. Two freely suspended nickel wires function as temperature sensing elements and are placed symmetrically before and behind a thin film heater.

After dicing the wafer and removing the SU-8 sacrificial layer the chips with a size of $5 \times 5 \mathrm{~mm}^{2}$ are mounted on a TO-8 socket and connected electrically by wire bonding. The concept is shown in Fig. 5 . This socket is placed in a gas flow channel and characterized.

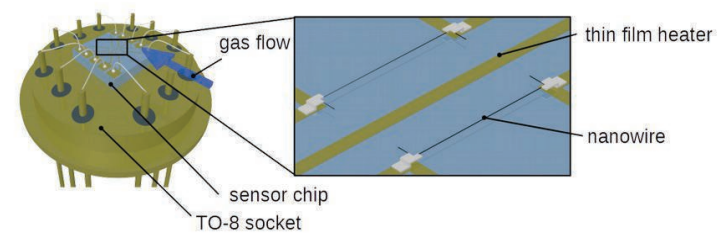

Fig. 5: Package of the calorimetric gas flow sensor chip. To characterize the chip it is mounted onto a TO-8 socket.

\section{Thermal coefficient of electrical resistance}

To determine the actual temperature of the wires upstream and downstream of the heating 
element the temperature dependent wire resistance has to be measured. Therefore it is necessary to determine the temperature coefficient $\alpha$ of the electrical resistance of the wires in order to estimate the actual temperature as follows:

$$
T=\frac{R-R_{0}}{R_{0} \cdot \alpha}+T_{0}
$$

With $R$ as the actual measured wire's resistance and $R_{0}$ as reference resistance at reference temperature $T_{0}$. To determine the temperature coefficient of the electrical resistance the sensor elements were placed in a climate chamber and the temperature was increased. The temperature coefficient $\alpha$ can be interpreted as the slope of the linear function (Eq. (2)).

$$
\frac{R-R_{0}}{R_{0}}=\alpha\left(T-T_{0}\right)
$$

The temperature was varied between $T_{0}=20^{\circ} \mathrm{C}$ (room temperature) and $T_{0}=60^{\circ} \mathrm{C}$. The mean and standard deviation of the measured values are plotted in Fig. 6. Two different wires were analyzed, an the results have only a maximal standard deviation of $\sigma_{R}=5 \cdot 10^{-4}$, which can hardly be shown in the plot.

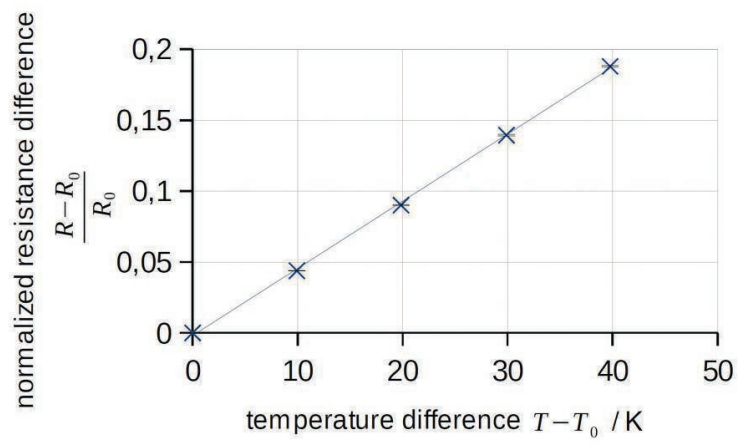

Fig. 6: Electrical resistance as a function of temperature

From the slope of the linear function the temperature coefficient $\alpha$ can be estimated as $\alpha=4,7 \cdot 10^{-3} 1 / \mathrm{K}$.

\section{Steady-state Characteristic}

The sensor elements have been characterized to determine their performance as calorimetric gas flow sensors. In the first step a steady state characterization was performed. The sensor chip is mounted onto a TO-8 socket, which is assembled into a gas flow channel. The channel with the integrated sensor is shown in Fig. 7.

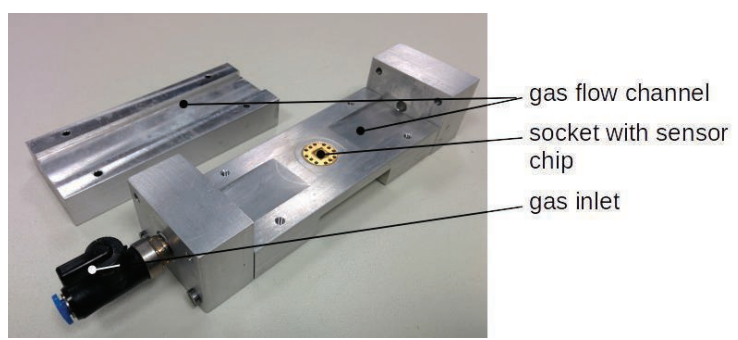

Fig. 7: Photograph of the channel used for steady state characterization

Then we apply a gas flow adjusted by a needle valve and a reference mass flow sensor (Honeywell AWM 3300). As test gas pressurized air is used.

Before the practical characterization we carried out a CFD simulation in order to determine the velocity and temperature field inside the channel. The simulation is necessary to find the optimal distance between the sensing wires and the heater which has been estimated to be about $300 \mu \mathrm{m}$. Tab. 3 shows the material parameters used for numerical calculations.

Tab. 3: Material parameters for numerical simulation

\begin{tabular}{|c|c|c|}
\hline dimension & unit & value \\
\hline kinematic viscosity (air) & $\frac{\mathrm{m}}{\mathrm{s}}$ & $10^{-5}$ \\
\hline density (air) & $\frac{\mathrm{kg}}{\mathrm{m}^{3}}$ & 1,2 \\
\hline $\begin{array}{l}\text { specific heat conduction } \\
\text { (brass socket) }\end{array}$ & $\frac{\mathrm{W}}{\mathrm{mK}}$ & 235 \\
\hline $\begin{array}{l}\text { specific heat conduction } \\
\text { (glass substrate) }\end{array}$ & $\frac{\mathrm{W}}{\mathrm{mK}}$ & 1,1 \\
\hline $\begin{array}{l}\text { heat diffusion } \\
\text { (brass socket) }\end{array}$ & $\frac{\mathrm{m}^{2}}{\mathrm{~s}}$ & $\underset{-5}{9,7 \cdot 10}$ \\
\hline $\begin{array}{l}\text { heat diffusion } \\
\text { (glass substrate) }\end{array}$ & $\frac{\mathrm{m}^{2}}{\mathrm{~s}}$ & $6 \cdot 10^{-7}$ \\
\hline heat power & $\mathrm{mW}$ & 150 \\
\hline
\end{tabular}
of the channel and the sensor element

The heater is driven at a constant power of $150 \mathrm{~mW}$. The resistance of the wires is captured by two digital multimeters (NI PXI 
4070 and NI PXI-4072) in four-wire mode. A schematic illustration of the setup is shown in Fig. 8.

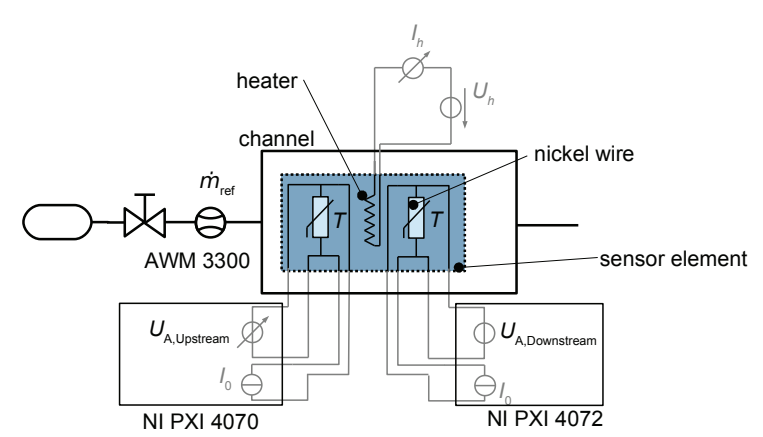

Fig. 8: Schematic of the setup for the steady state characterization of the sensor element

The measured resistances are converted into temperature values by eqn. (2).

A comparison of measured and simulated values is shown in Fig. 9. It can bee seen that simulated and measured values coincide very well. The slight differences are due to the neglection of the wires dimensions and the real air pressure during the simulation.

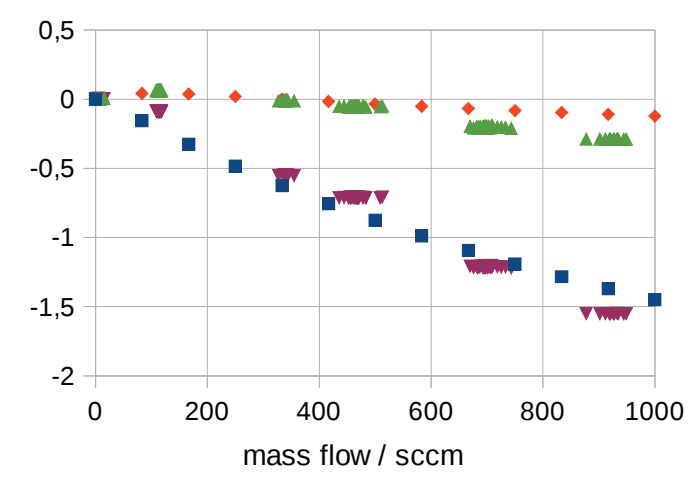

- Downstream, simulated $\Delta$ Downstream, measured

$\checkmark$ Upstream, measured Upstream, simulated

Fig. 9: Simulated and measured temperature of the down- and upstream nickel wires

\section{Conclusion and outlook}

A process for the fabrication of very thin, freely suspended nickel wires have been developed. A calorimetric gas flow sensor using these wires as sensitive elements has been fabricated and the functionality have been shown. In addition a CFD calculation was performed to determine the sensors performance.

The next step is to perform a transient characterization of the sensor in order to demonstrate the potential for an extremely small response time of the device.

\section{Acknowledgment}

This work was supported in part by the German Federal Ministry of Education and Research (BMBF) under the grant 16 SV5476.

\section{Literature}

[1] N. . Nguyen, "Micromachined flow sensors -a review," Flow Meas. Instrum., vol. 8, no. 1, pp. 7-16, März 1997.

[2] M. Elwenspoek, "Thermal flow micro sensors," in CAS, 1999, vol. 2, pp. 423435.

[3] Y.-H. Wang, C.-P. Chen, C.-M. Chang, C.-P. Lin, C.-H. Lin, L.-M. Fu, and C.-Y. Lee, "MEMS-based gas flow sensors," Microfluid. Nanofluidics, vol. 6, no. 3, pp. 333-346, Mar. 2009.

[4] J. Chen, Jun Zou, and Chang Liu, "A surface micromachined, out-of-plane anemometer," presented at the Micro Electro Mechanical Systems, 2002. The Fifteenth IEEE International Conference on, 2002, pp. 332-335.

[5] K. Jasek, T. Budzynski, and E. Stryszak, "Optopneumatic detector with platinum flow sensor," Acta Phys. Pol.-Ser. Gen. Phys., vol. 116, no. 3, p. 321, 2009.

[6] H.-E. de Bree, P. Leussink, T. Korthorst, H. Jansen, T. S. J. Lammerink, and M. Elwenspoek, "The $\mu$-flown: a novel device for measuring acoustic flows," Sens. Actuators Phys., vol. 54, no. 1-3, pp. 552557, Jun. 1996.

[7] E. J. Menke, M. A. Thompson, C. Xiang, L. C. Yang, and R. M. Penner, "Lithographically patterned nanowire electrodeposition," Nat. Mater., vol. 5, no. 11, pp. 914-919, Nov. 2006. 